\title{
Epithelial cells within the human pancreas do not coexpress mesenchymal antigens: epithelial-mesenchymal transition is an artifact of cell culture
}

\author{
Karen L Seeberger ${ }^{1}$, Alana Eshpeter ${ }^{1}$, Ray V Rajotte ${ }^{1,2,3}$ and Gregory S Korbutt ${ }^{1,2}$
}

Pancreatic mesenchymal stem cells (MSCs) may be derived from human $\beta$-cells undergoing reversible epithelial-mesenchymal transition (EMT), suggesting that they could be a potential source of new $\beta$-cells. In this study we sought to determine the origin of pancreatic MSCs in the nonendocrine pancreas. Double immunofluorescent (IF) staining and flow cytometry were used to assess the cell phenotype of nonendocrine pancreas tissue following islet procurement, during in vitro expansion of MSCs, and after differentiation. IF staining of paraffin-embedded pancreatic biopsy sections was used to assess cell phenotype in vivo. In this study we demonstrated that: (1) pancreatic epithelial cells do not express MSC antigens in vivo; (2) following islet isolation EpCAM- and CK19-positive epithelial cells coexpressed the MSC antigens CD44 (32 $\pm 8 \%$ and $38 \pm 10 \%)$ and CD29 (85 $\pm 4 \%$ and $64 \pm 4 \%$ ); (3) during in vitro expansion the number of single-positive epithelial and double-positive epithelial/MSCs decreased whereas the number of single-positive MSCs increased and (4) differentiated MSCs do not revert to a true epithelial cell phenotype in our culture conditions, as epithelial cell surface markers (EpCAM, CK19 and E-Cadherin) are not reexpressed, although the MSC phenotype is altered. This study demonstrates that MSCs may be derived in vitro via a pancreatic epithelial cell undergoing EMT, however it is more likely that a small percentage of MSCs that reside in the adult pancreas are proliferating whereas the epithelial cells are negatively selected by the experimental culture conditions.

Laboratory Investigation (2009) 89, 110-121; doi:10.1038/labinvest.2008.122; published online 15 December 2008

KEYWORDS: $\beta$-cells; mesenchymal stem cells; epithelial-mesenchymal transition and differentiation

Islet transplantation requires a minimally invasive procedure and has been shown to ameliorate diabetes by eliminating the need for daily insulin injections. Prolonged islet graft survival does however require chronic immunosuppression that may have detrimental side effects, and moreover only $10 \%$ of recipients remain insulin independent at 5 years after transplant. ${ }^{1}$ Nonetheless, these patients remain C-peptide-positive and have improved glycemic control. Possible reasons for the loss of graft function in these patients include toxicity of the immunosuppressive drugs, chronic allograft rejection, recurrence of autoimmunity, lack of islet precursors in the graft to allow for continuous $\beta$-cell turnover ${ }^{2}$ and the limited longevity of an adult islet graft. ${ }^{3}$ Concurrently, the shortage of human pancreases limits the availability of donor tissue, which precludes islet transplantation from becoming a more applicable form of therapy.

The expansion of $\beta$-cells from a progenitor/stem cell source is a practical alternative to the shortage of donor islets. Several studies have reported that progenitors reside within the pancreas itself. Possible sources of these progenitors are the pancreatic ductal epithelium, ${ }^{4-11}$ acinar tissue, ${ }^{12-14}$ islets $^{15,16}$ and mesenchyme. ${ }^{17-23}$ Studies have indicated that mesenchymal stem cells (MSCs) are multipotent and capable of deriving functional endoderm. ${ }^{24-29}$ Although MSCs isolated from bone marrow can be differentiated into insulinproducing cells ${ }^{28,29}$ these newly derived $\beta$-cells secrete significantly lower amounts of insulin as compared to native adult $\beta$-cells. ${ }^{28,29}$ As $\beta$-cells could be derived from cells of

\footnotetext{
${ }^{1}$ Alberta Diabetes Institute, University of Alberta, Edmonton, $A B$, Canada; ${ }^{2}$ Department of Surgery, University of Alberta, Edmonton, $A B$, Canada and ${ }^{3}$ Department of Medicine, University of Alberta, Edmonton, AB, Canada

Correspondence: Dr GS Korbutt, PhD, Department of Surgery, Alberta Diabetes Institute, University of Alberta, 5-002 HRIF East, Edmonton, AB, Canada T6G 2E1. E-mail: korbutt@ualberta.ca

Received 30 July 2008; revised 5 September 2008; accepted 9 September 2008
} 
mesenchymal origin, ${ }^{26,28,29}$ we hypothesized that MSCs originating from the pancreas would possess a greater potential to differentiate into functional $\beta$-cells as compared to MSCs isolated from nonpancreatic tissues. We previously reported that MSCs could be isolated and expanded in large numbers from human pancreatic ductal epithelium. ${ }^{20}$ These pancreatic MSCs were phenotypically similar to bone marrow-derived MSCs as they expressed typical MSC cell-surface antigens and could be differentiated in vitro into mesoderm. Moreover, we demonstrated that pancreatic MSCs were multipotent as they were able to cross the mesodermal lineage and derive cells of endoderm origin such as hepatocytes. ${ }^{20}$ Our attempts to differentiate pancreatic MSCs into functional $\beta$-cells resulted in the expression of some genes necessary for islet and $\beta$-cell development including NeuroD, PDX-1, ngn3 and Pax4 in addition to several endocrine hormone transcripts; however, we have not observed protein expression of these gene products. Although we first reported that pancreatic-derived MSCs lacked insulin protein expression, ${ }^{20}$ several others have reported that isletlike clusters derived from MSCs or mesenchymal cell intermediates secrete insulin, C-peptide and glucagon. ${ }^{18,21-23}$ One model in particular suggested that MSCs were derived via human $\beta$-cells undergoing a reversible epithelial-mesenchymal transition (EMT). ${ }^{18}$ However, this mechanism for deriving new islets has become controversial. ${ }^{30-33}$ Reports that use lineage tracing of $\beta$-cells from transgenic mice concluded that in culture, MSCs were not derived from $\beta$-cells but instead preexist within the adult islet. ${ }^{31,32}$ However, in a recent report, Russ $e t a l^{34}$ successfully labeled human $\beta$-cells using a dual lentivirus system and demonstrated that unlike mouse $\beta$-cells, human $\beta$-cells do dedifferentiate and can be expanded in culture. Despite this recent progress the origin of human pancreatic MSCs from cultured nonendocrine pancreas tissue is undetermined.

The objective of this study was to determine the origin of human pancreatic MSCs from cultured nonendocrine pancreatic tissue. Similar to MSCs derived from purified islets, MSCs from the nonendocrine pancreas may be derived from an epithelial cell within the acinar tissue that undergoes an EMT, ${ }^{18}$ or from preexisting MSCs that reside within the exocrine and/or ductal epithelium. ${ }^{31,32}$ Determining from which population pancreatic MSCs are derived should enable future studies to either positively select these cells or discard certain populations to enhance the isolation of the elusive $\beta$-cell progenitor. In this study, we further characterized cell cultures of human pancreatic-derived MSCs with respect to the epithelial cell content, MSC content and the coexpression of MSC and epithelial cell antigens, and compared this to the expression in the native pancreas and differentiated pancreatic MSCs. We demonstrated that: (1) native pancreatic epithelial cells do not coexpress typical MSC antigens; (2) in 2-day cultures of human pancreatic digests EpCAM- and CK19-positive epithelial cells express the MSC antigens CD44 (32 $\pm 8 \%$ and $38 \pm 10 \%$, respectively) and
CD29 (85 $\pm 4 \%$ and $64 \pm 4 \%$, respectively); (3) during in vitro expansion of pancreatic-derived MSCs the proportion of single-positive epithelial and double-positive epithelial/MSCs decreases; and more importantly we demonstrated that (4) differentiated MSCs do not revert to a true epithelial cell phenotype in our culture conditions, as epithelial cellsurface markers (EpCAM, CK19 and E-Cadherin) are not reexpressed, although the MSC phenotype is altered.

\section{MATERIALS AND METHODS}

Nonendocrine pancreatic digests from five independent human donors ranging from 43 to 65 years of age were obtained following islet purification and cultured as described previously. ${ }^{20}$ Briefly, the islet-depleted tissue was cultured for 2-4 days in RPMI medium (Invitrogen, Burlington, Canada), supplemented with $10 \%$ fetal bovine serum (Invitrogen) to produce cellular aggregates, composed primarily of ductal epithelium. The resulting cellular aggregates were then used to derive and expand MSCs. Ductal aggregates were dissociated with $0.1 \%$ trypsin (SigmaAldrich, Oakville, Canada) and single cells were fixed for flow cytometry analysis, preserved for RNA extraction and RT-PCR as described previously, ${ }^{20}$ and seeded into either $150 \mathrm{~mm}$ tissue culture-treated plates or 4- to 8-well tissue culture-treated chamber slides (BD Biosciences, Bedford, MA, USA). Human pancreas biopsy sections were fixed in Z-fix (Anatech Ltd, Battle Creek, MI, USA) and paraffin embedded.

\section{Cell Expansion and Differentiation}

Single cells were cultured and expanded on $150 \mathrm{~mm}$ tissue culture-treated dishes and 4- to 8-well chamber slides utilizing RPMI 1640 supplemented with 10\% fetal bovine serum, $10 \mathrm{mM}$ HEPES, $1 \mathrm{mM}$ sodium pyruvate, $71.5 \mu \mathrm{M}$ $\beta$-mercaptoethanol and $20 \mathrm{ng} / \mathrm{ml}$ each of EGF and bFGF (Invitrogen) in conditions described previously. ${ }^{20}$ To assess changes to cell phenotype during this in vitro expansion, cells in chamber slides were harvested and fixed with $1 \%$ formaldehyde (BDH Laboratory Supplies, UK) after 24, 48 and $72 \mathrm{~h}$ of culture. Cells grown in monolayers on $150 \mathrm{~mm}$ dishes were detached with trypsin-EDTA and replated after achieving confluence for up to four passages (30 days). Pancreatic MSCs were differentiated as a monolayer utilizing a serum-free multistep, 15-day protocol followed by cellular aggregation. ${ }^{20}$ In step 1, the cell monolayer was treated for 3 days with Iscove's Modified Dulbecco's Medium (Invitrogen). In step 2, the medium was supplemented with $20 \mathrm{ng} / \mathrm{ml}$ of Oncostatin M (Cedarlane, Hornby, Canada), $1 \mu \mathrm{M}$ dexamethasone (Sigma-Aldrich), and $50 \mathrm{mg} / \mathrm{ml}$ of ITS + Premix (BD Biosciences) for 3-6 days. In steps 3 and 4 , the medium was supplemented with $10 \mathrm{mM}$ nicotinamide (Sigma-Aldrich) for 3 days followed by 3 days with $10 \mathrm{mM}$ nicotinamide and $10 \mathrm{nM}$ of exendin-4 (Sigma-Aldrich). In step $5,10 \mathrm{ng} / \mathrm{ml}$ of transforming growth factor- $\beta 1$ (TGF- $\beta 1$; Cedarlane) was included with nicotinamide and exendin-4 
for 2-3 days. In step 6, cells were detached with trypsin and aggregated by reconstituting cells at 125000 cells $/ \mathrm{ml}$ in IMDM supplemented with exendin-4, nicotinamide, TGF$\beta 1$, ITS + Premix in ultra low attachment plates (Corning, NY, USA) for 3 days.

\section{Double Immunofluorescent Staining}

Immunofluorescence (IF) analysis was performed on paraffin-embedded adult pancreatic sections and on cells expanded on chamber slides after fixation with $1 \%$ formaldehyde. Paraffin sections were rehydrated and immersed in Tris-EDTA for antigen retrieval by microwaving. Paraffin sections and chamber slides were permeabilized for $3 \mathrm{~min}$ with $0.1 \%$ Triton X-100 and blocked with $20 \%$ normal goat serum (NGS) in PBS for $1 \mathrm{~h}$. If biotinylated primary antibodies were used, an avidin/biotin (Vector Laboratories Inc., Burlingame, CA, USA) blocking step was included before the addition of the first primary antibody. Primary antibodies were diluted in 5\% NGS at the following concentrations: 1:250 rabbit anti-amylase (Sigma), 1:50 mouse anti-CK19 (Dako, Mississauga, Canada), 1:50 mouse anti-CD29 biotinylated (R\&D, Minneapolis, MN), 1:100 mouse antiE-Cadherin (R\&D), 1:200 mouse anti-EpCAM (Stem Cell Technologies, Vancouver, Canada), 1:5000 guinea pig antiglucagon (Cedarlane), 1:1000 guinea pig anti-insulin (Dako) and (1:200) mouse anti-vimentin biotinylated (Lab Vision, Fremont, CA, USA). The secondary antibody was either a Streptavidin AlexaFluor antibody (1:200) or an appropriate species-specific AlexaFluor conjugate (Molecular Probes, Eugene, OR, USA). Before the addition of the second primary antibody a second blocking step was included using 1:10 Fab fragment goat anti-mouse IgG in 5\% NGS (Jackson ImmunoResearch Laboratories Inc., West Grove, PA, USA). Following the second primary/secondary antibody step slides were coverslipped with ProLong Gold anti-fade reagent with DAPI (Invitrogen) to counter stain nuclei and preserve fluorescence. Primary antibodies raised in mouse were never used in combination unless one primary was biotinylated and multiple blocking steps were included. Negative controls were sections and chamber slide wells incubated without primary antibodies. All slides were visualized with an Axioscope II equipped with AxioCam MRC and analyzed with Axiovision 4.6 (Carl Zeiss, Gottingen, Germany).

\section{Flow Cytometry}

Cell-surface antigen expression at the start of the study, after 2-4 days culture, MSC expansion and differentiation were also determined by flow cytometry analysis as described previously. ${ }^{20}$ Briefly, cells were dissociated, followed by fixation with $1 \%$ formaldehyde, washed with PBS and permeabilized by $0.3 \%$ saponin for $10 \mathrm{~min}$ at room temperature when required for intracellular markers. Unlabeled primary antibodies were applied for $30-60 \mathrm{~min}$ at $4^{\circ} \mathrm{C}$ followed by washes and incubation with fluorescent secondary antibodies. For double staining, the unconjugated antibody was incubated first, followed by incubation with fluorescent secondary and conjugated primary antibodies. When staining for both cell-surface and intracellular markers in the same sample, involving one unconjugated primary antibody and one directly conjugated antibody, both raised in mouse, the unconjugated antibody was applied first, then its companion fluorescent secondary antibody, followed by a combination permeabilization and blocking step using Fab goat antimouse IgG (Jackson ImmunoResearch) at room temperature for $15 \mathrm{~min}$. The cells were then incubated with the directly conjugated intracellular antibody. Before analysis, all cells were washed twice with PBS, and analyzed on a FACS Calibur (BD Biosciences, Mississauga, Canada) using Cell Quest Pro software and compared to matched isotype controls. In addition to the antibodies used to confirm MSC phenotype, ${ }^{20}$ antibodies to endocrine and exocrine hormones as well as epithelial cell antigens were used: 1:500 guinea pig anti-insulin (Dako), 1:5000 guinea pig anti-glucagon (Sigma), 1:250 rabbit anti-amylase (Sigma), 1:50 mouse antiCK19 (Dako), 1:100 mouse anti-E-Cadherin (R\&D) and 1:200 mouse anti-EpCAM (Stem Cell Technologies). Values are expressed as mean percent \pm s.e.m.

\section{RESULTS \\ Human Pancreatic Epithelial Cells do not Coexpress MSC Antigens In Vivo}

To determine if epithelial cells within the adult pancreas coexpress MSC cell markers, we analyzed pancreatic biopsy sections $(n=5)$ by double IF staining. We chose to assess the epithelial markers CK19, E-Cadherin and EpCAM along with antibodies we have used to characterize pancreatic MSCs (CD29, CD44 and vimentin). Before determining if epithelial cells that express E-Cadherin and EpCAM also coexpress MSC cell-surface markers, we first determined where E-Cadherin- and EpCAM-positive epithelial cells were localized in the pancreas by costaining with insulin, glucagon and amylase. E-Cadherin expression was confined within the $\mathrm{i}$ slets (Figure 1a, d and g), and colocalized with insulinpositive $\beta$-cells (Figure 1c), and not colocalized with glucagonor amylase-positive cells (Figure if and i). EpCAM was expressed throughout the pancreas and not colocalized to a specific cell population (Figure 2). Double IF staining of pancreatic sections demonstrated that EpCAM-, E-Cadherinand CK19-positive epithelial cells (Figures 3b, e, h, 4b, e and h) do not coexpress MSC antigens (vimentin and CD29; Figures $3 c, f, i, 4 c, f$ and i). Vimentin staining was present throughout the connective tissue (mesenchyme derived) and in the vascular lining and ductal walls (Figure 3a, d and g). CD29 expression was found within the vascular lining and ducts of the pancreas (Figure $4 \mathrm{a}, \mathrm{d}$ and g). More specifically vimentinand CD29-positive cells were located in the outer wall of the ducts whereas CK19-positive cells lined the lumen (Figures 3i and 4i). CD44 staining was similar to that observed for vimentin (data not shown). 

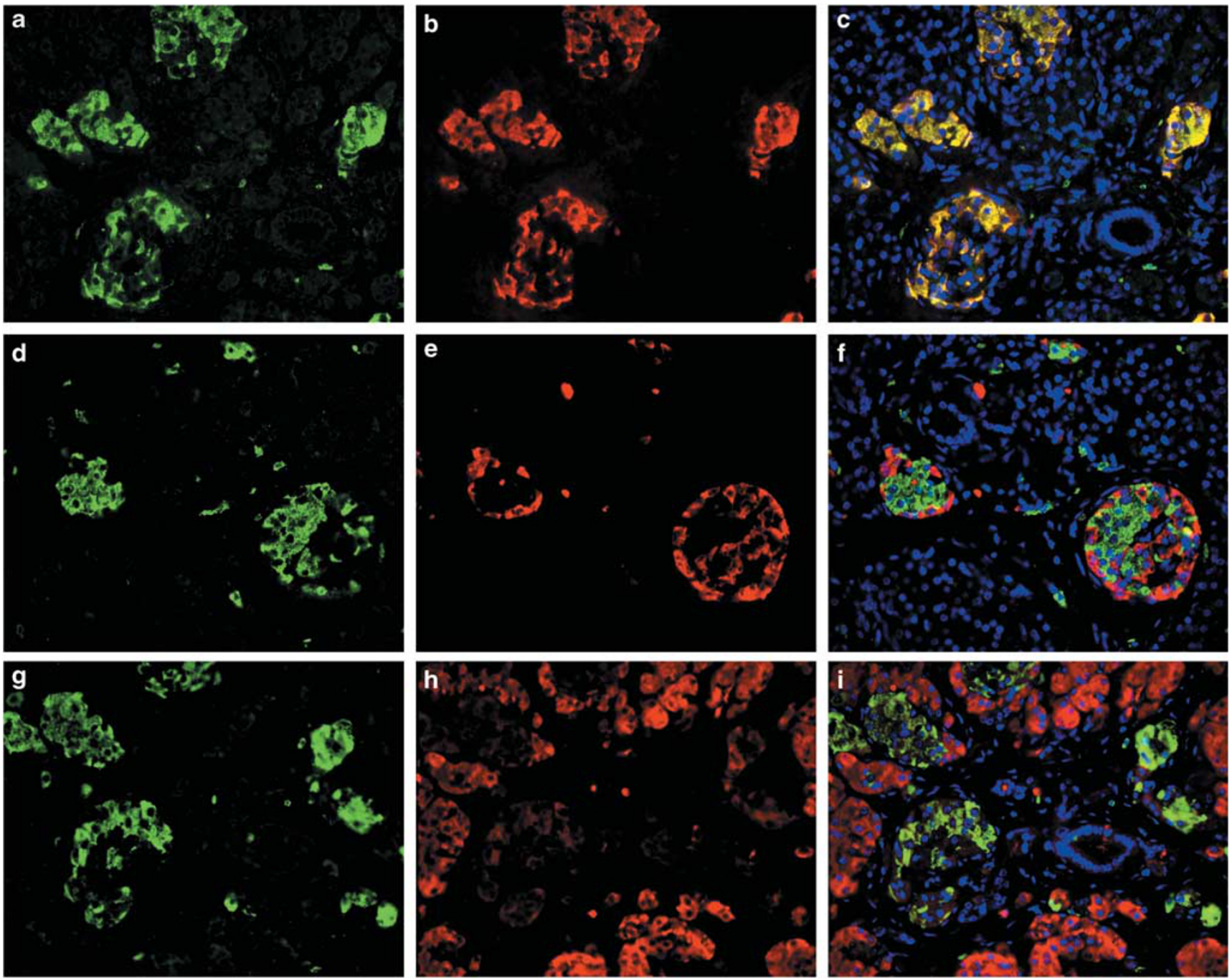

Figure 1 Double IF staining of adult human pancreas section. E-Cadherin (green; $\mathbf{a}, \mathbf{d}, \mathbf{g}$ ), insulin (red; $\mathbf{b}$ ), glucagon (red; e) and amylase (red; $\mathbf{h}$ ) demonstrate that E-Cadherin is expressed in $\beta$-cells (yellow; $\mathbf{c}$ merged) but not $\alpha$-cells ( $\mathbf{f}$ merged) or amylase-positive cells (i merged). Magnification is $400 \times$.

\section{Characterization of Pancreatic-Derived Cell Populations}

Similar to our previous study, ${ }^{20}$ we expanded MSCs from the nonendocrine pancreatic tissue following islet purification and 2 days of culture, and confirmed their cellular phenotype by assessing the expression of a number of cell-surface antigens commonly used to characterize bone marrow-derived MSCs. To determine if pancreatic MSCs were derived from epithelial cells within the nonendocrine pancreas that may possibly transdifferentiate and/or undergo a reversible EMT, we characterized our starting cell populations (2-day cultures), following tissue culture for $24,48,72 \mathrm{~h}$ and at the first (7-10 days) and second (14-20 days) passages by flow cytometry. As described previously, ${ }^{20}$ our starting cell preparations were positive for insulin $(4 \pm 1.82 \%)$, glucagon (3.8 $\pm 2.3 \%)$, amylase $(45.2 \pm 11 \%)$ and CK19 (62.2 $\pm 4.29 \%$; $n=5)$. The flow cytometry histograms in Figure 5a from a single donor comparing the starting cell fraction with cells expanded for 2 weeks demonstrated that following islet purification and 2 days of culture the starting cell composition consisted primarily of epithelial cells that were EpCAM (99\%), CK19 (68\%) and amylase (61\%) positive as well as cells positive for CD29 (90\%) and CD44 (50\%). In contrast, these initial cell preparations were less than $4 \%$ insulin- and E-Cadherin-positive (Figure 5a), thereby indicating that all $\beta$-cells expressed E-Cadherin. As these cells were cultured, the proportion of cells expressing typical MSC antigens (vimentin, CD105, CD90, CD13, CD29 and CD44) increased whereas the percentage of epithelial cells (CK19 and EpCAM) decreased (Figure 5b).

When determining the percentage of epithelial cells that coexpressed MSC antigens, we observed that in the starting preparation a large proportion of EpCAM- and CK19-positive epithelial cells coexpressed MSC antigens (Figure 6). In this fraction, EpCAM- and CK19-positive cells coexpressed CD29 $(85.4 \pm 3.97 \%$ and $64 \pm 4.05 \%$, respectively). EpCAM- and CK19-positive cells at this time point 

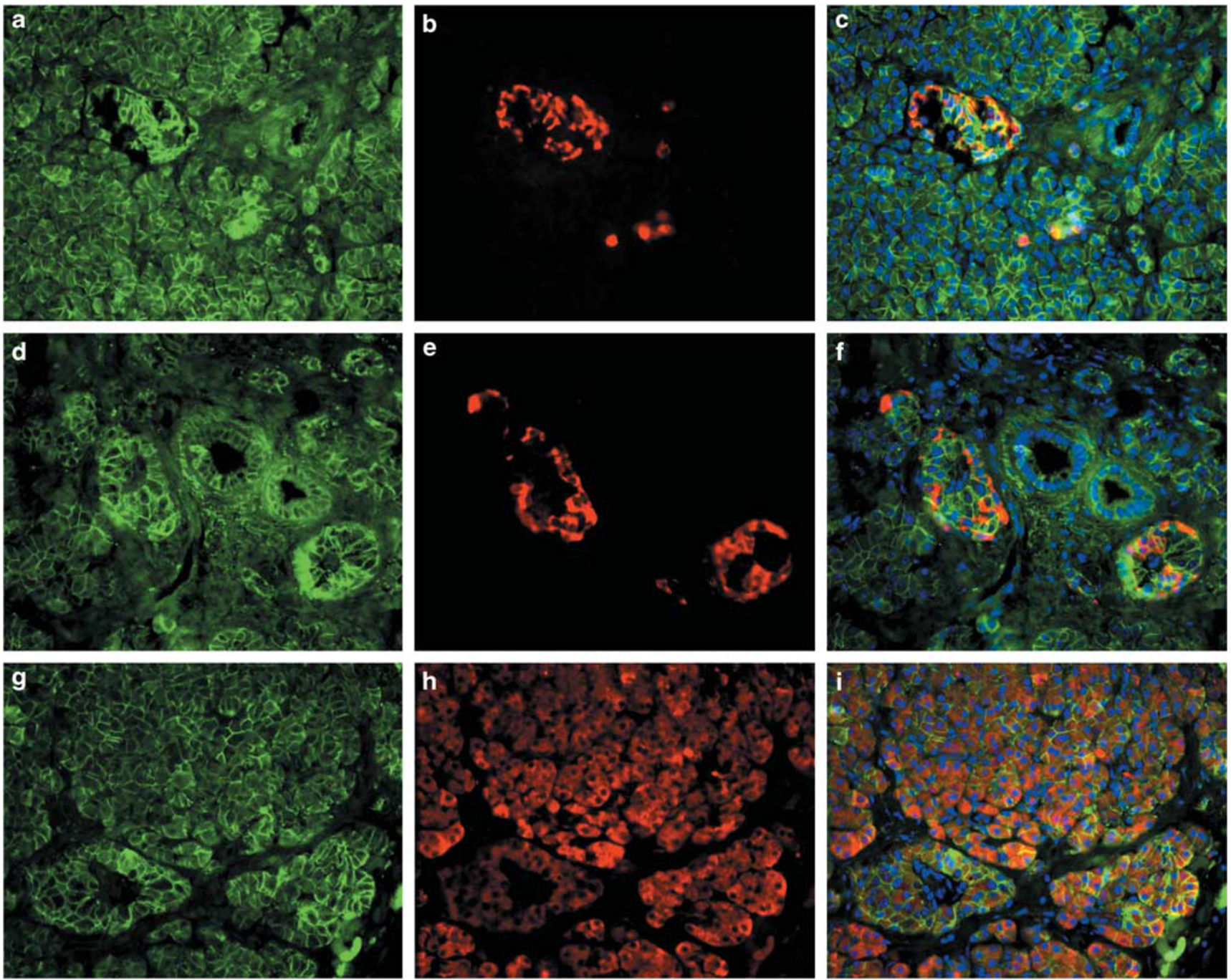

Figure 2 Double IF staining of adult human pancreas section. EpCAM is expressed throughout the pancreas (a, $\mathbf{d}, \mathbf{g})$. EpCAM is expressed on the surface of $\beta$-cells (c merged) stained with insulin (b), $\alpha$-cells (f merged) stained with glucagon (e) and exocrine cells (i merged) stained with amylase (h). Magnification is $400 \times$.

also expressed CD44 but at a much lower level $(32.2 \pm 8.18 \%$ and $38.6 \pm 10.01 \%$, respectively). Vimentin was also coexpressed (Figure 6) by these epithelial cells $(20.2 \pm 5.35 \%$ and $9.0 \pm 1.26$, respectively) but at a significantly lower proportion because the percentage of vimentin-positive cells in the starting cell fraction was less than $20 \%$ (Figure 5b). We also observed that the proportion of epithelial cells that coexpressed CD44 and CD29 decreased with each passage (Figure 6). By contrast, the cells that coexpressed vimentin were highest at passage 1 , reflective of the fact that the proportion of vimentin-positive cells increased with culture. In these cell fractions there were also single-positive epithelial (EpCAM and CK-19) cells, and cells only expressing the typical MSC antigens.

The starting cell preparations after 2 days in culture were also dissociated, cultured on 4- and 8-well chamber slides (24, 48 and $72 \mathrm{~h}$ ) and assessed for CD29 (Figure 7a) and vimentin (Figure 7e) expression, and costained with EpCAM (Figure 7) and CK19 (data not shown). Double IF analysis correlated with flow cytometry observations as epithelial cells positive for EpCAM (Figure $7 b$ and $\mathrm{f}$ ) also coexpressed CD29 (Figure 7c) and vimentin (Figure 7g). Corresponding scatter plots from the same time point collected from cells expanded on $150 \mathrm{~mm}$ tissue culture treated dishes demonstrated that $98 \%$ of the cells coexpressed EpCAM and CD29 (Figure 7d), and 39\% coexpressed vimentin (Figure $7 \mathrm{~h}$ ). Within the $72 \mathrm{~h}$ time frame similar results were observed at 24 and $72 \mathrm{~h}$ (data not shown).

\section{Epithelial Cell Phenotype is not Restored following $\beta$-Cell Differentiation}

Using previously developed protocols ${ }^{20}$ to try to differentiate these pancreatic MSCs into islet cells we were unable to achieve expression of endocrine proteins, but we observed 

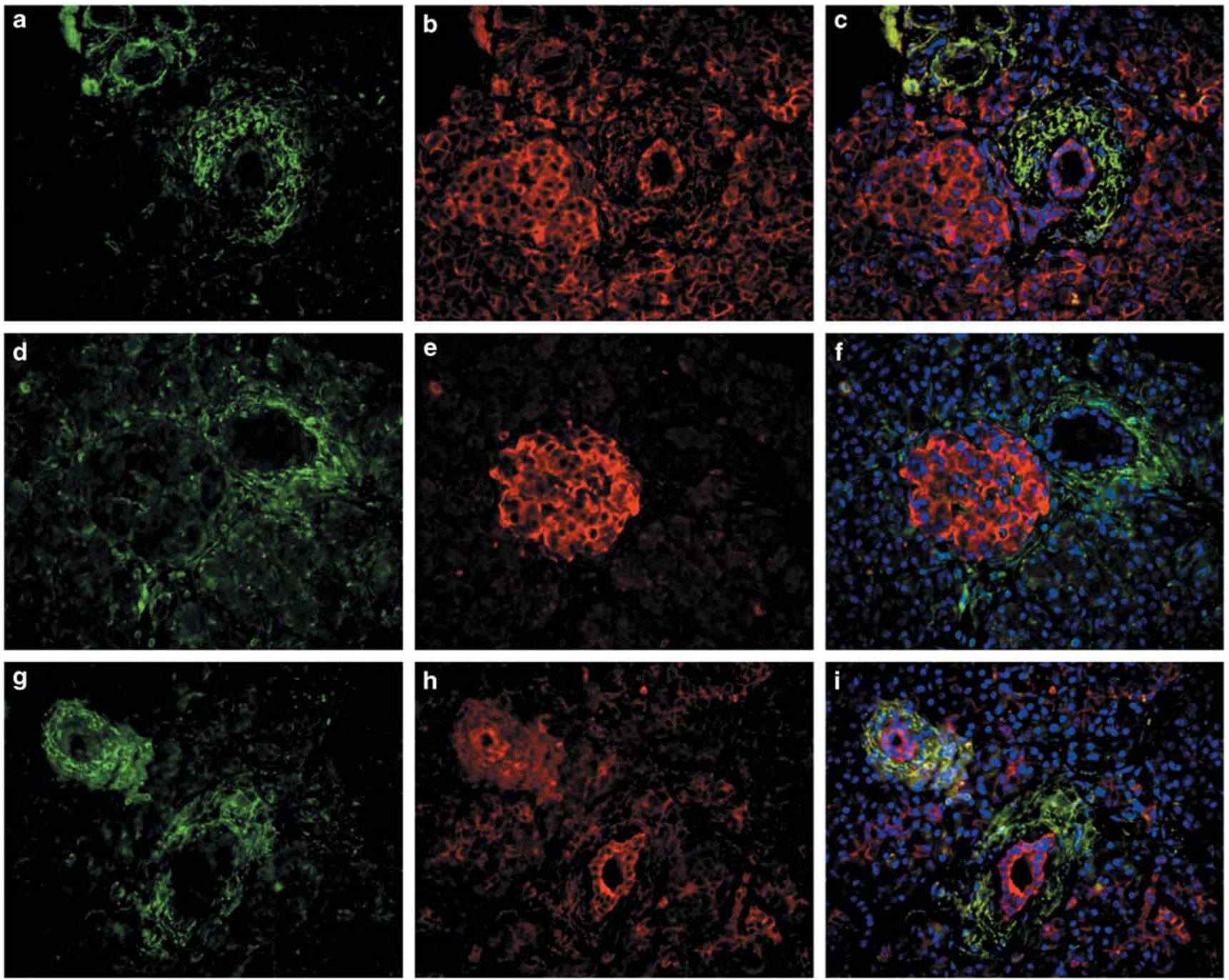

Figure 3 Representative pancreatic section stained by double IF. Vimentin (green; a, d, g), EpCAM (b), E-Cadherin (e) and CK19 (h) staining demonstrate that vimentin is not coexpressed in adult pancreatic epithelial cells (c, $\mathbf{f}, \mathbf{i}$ merged). Magnification is $400 \times$.

that the cell phenotype was altered. When we compared the cell-surface antigen expression of differentiated cells to undifferentiated cells by flow cytometry, we observed a decrease in the expression of MSC antigens in the differentiated cells. In the differentiated cells, CD44 expression was reduced to $2.4 \pm 0.98 \%$ from $87.8 \pm 7.1 \%$, CD105 was reduced to $72.2 \pm 15.2 \%$ from $98.0 \pm 0.0 \%, \quad$ CD 90 was reduced to $73.6 \pm 12.3 \%$ from $80.6 \pm 12.2 \%, \mathrm{CD} 13$ was reduced to $67.4 \pm 11.8 \%$ from $78.2 \pm 5.4 \%$, CD29 was reduced to $86.4 \pm 7.7 \%$ from $98.0 \pm 0.0 \%$ and vimentin was reduced to $94.8 \pm 1.1 \%$ from $98.2 \pm 0.2 \%$. The histograms (Figure 8 ) from a single donor show the decreased antigen expression following differentiation compared with undifferentiated cells cultured for the same length of time (18 days). More importantly cells differentiated with this protocol did not express or reexpress epithelial (CK19, EpCAM and E-Cadherin) cell antigens (Figure 8) or endocrine hormones (data not shown).

\section{DISCUSSION}

Several studies have reported the in vitro expansion of multipotent MSCs from pancreatic islet cultures and the differentiation of these MSCs into insulin expressing cells. ${ }^{17-19,21-23}$ These studies suggest that MSCs either originate from $\beta$-cells themselves that undergo a reversible EMT to generate new $\beta$-cells ${ }^{18}$ or reside within islets as a distinct cell population. ${ }^{21-23}$ Whether MSCs are derived via EMT or are present within islets, the majority of these studies report increased levels of insulin mRNA transcripts following differentiation but the transcript levels are less than $1 \%$ of human islets. ${ }^{18,22,23}$ Others have demonstrated cellular insulin expression in differentiated cells; however these cells remain unresponsive to a glucose challenge. ${ }^{21,22} \mathrm{Few}$ studies have convincingly demonstrated that these differentiated cells are truly functional $\beta$-cells that also express epithelial cell antigens. ${ }^{18,23}$ Therefore, the origin and potential of MSCs derived from islet cultures in the generation of functional insulin secreting $\beta$-cells remains controversial. 

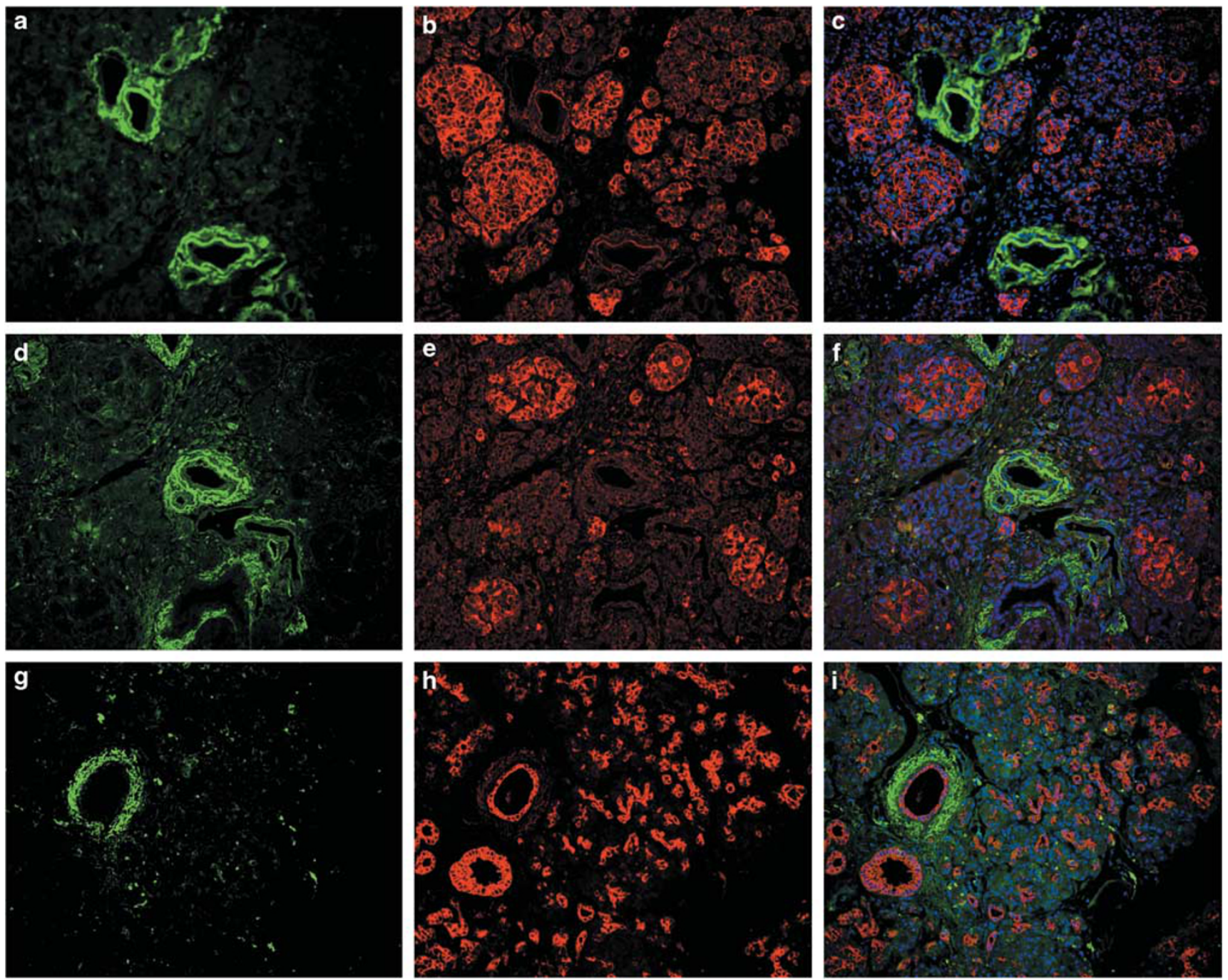

Figure 4 Representative pancreatic section stained by double IF. CD29 (green; a, d, g) EpCAM (b), E-Cadherin (e) and CK19 (h) staining demonstrates that CD29 is not expressed in pancreatic epithelial cells (c, $\mathbf{f}, \mathbf{i}$ merged). Magnification is $200 \times$.

To further define the role of pancreatic-derived MSCs, we sought to determine where these cells originate in cultured nonendocrine pancreatic tissue and whether they are capable of differentiating into functional pancreatic epithelial cells that express the most fundamental epithelial cell antigens. Previously we demonstrated that MSCs could be isolated and expanded in large numbers from human pancreatic ductal epithelium. ${ }^{20}$ Our attempts to differentiate these MSCs into functional $\beta$-cells resulted in the expression of some genes necessary for islet and $\beta$-cell development and endocrine hormone transcripts; however these cells did not express cellular insulin. ${ }^{20}$

With respect to where in the nonendocrine pancreas MSCs originate, we demonstrated that 2-day cultures of pancreatic digests almost void of endocrine cells $(4 \pm 1.82 \%$ insulin $)$ contained single-positive MSCs and epithelial cells, as well as epithelial cells that coexpressed MSC antigens. In addition, we show that during in vitro expansion of pancreatic MSCs the epithelial cell population decreased over time regardless if
MSC antigens were coexpressed. We also observed that the cellular composition of MSCs in the initial 2-day cultured digest was quite different compared to the cells expanded for 7-14 days (first passage). A subpopulation of MSCs were present in the starting preparation (2-day cultures) because only CD29 and CD44 were expressed by a large proportion of cells at this time point (Figure $5 \mathrm{a}$ and $\mathrm{b}$ ) thereby indicating that pancreatic MSCs are heterogeneous. By contrast, pancreatic sections showed no evidence of epithelial cells that coexpressed MSC antigens. This observation was expected because in normal, differentiated tissue there should not be a loss of epithelial phenotype whereas in tissue exposed to collagenase digestion and 2-day culture this phenomenon could easily occur. Therefore, MSCs in these pancreatic cultures may have originated from MSCs that initially preexist such as the vimentin-positive cells observed in pancreatic sections (Figure 3) and/or possibly from epithelial cells that dedifferentiated into MSCs (ie, EMT). 

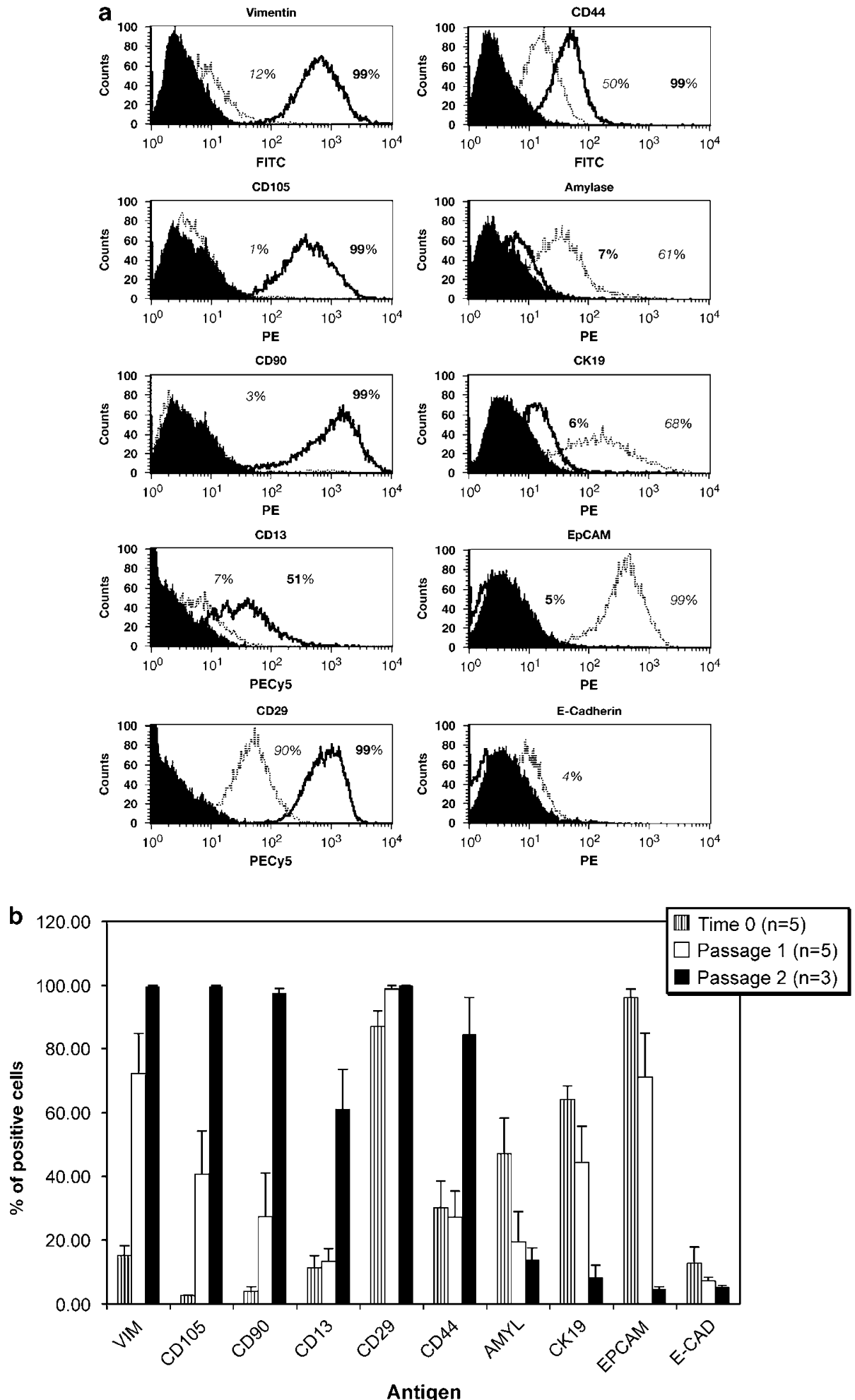

Figure 5 (a) Representative MSC antigen profile of nonendocrine starting tissue compared with MSCs expanded in vitro (14 days). The starting tissue (solid gray line, italic script) is comprised mainly of epithelial cells (EpCAM and CK19), CD44 and CD29 positive cells. Vimentin, CD105, CD90 and CD13 are equal to or less than $12 \%$. During expansion (solid black line, bold script), the MSC fraction increases whereas the epithelial component decreases. Solid histogram denotes isotype control. (b) During in vitro expansion the percent of single-positive epithelial cells (amylase, CK19, EpCAM and E-Cadherin) decreases whereas the percent of single-positive MSCs (vimentin, CD105, CD90, CD13, CD29, CD44) increases. Values are mean percent \pm s.e.m. 

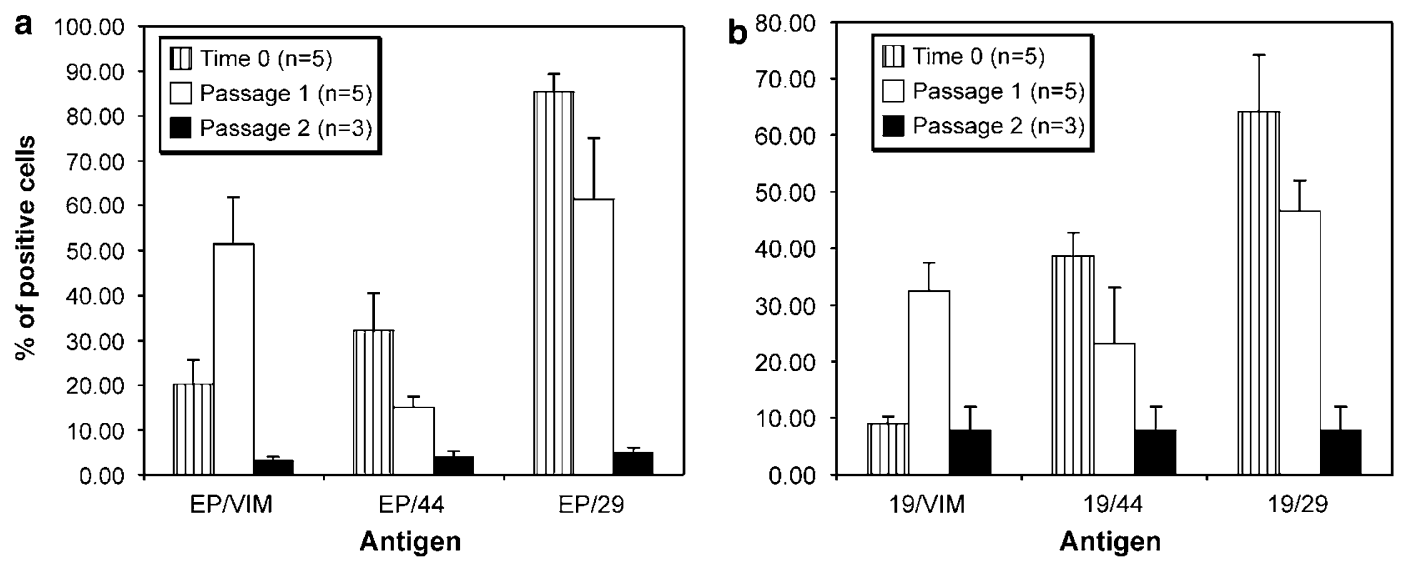

Figure 6 During in vitro expansion, the percent of epithelial cells that coexpress MSC antigens decrease. The percent of epithelial cells (EpCAM (a) and CK19 (b)) that coexpress MSC antigens (CD44 and CD29) is highest in the starting tissue following islet harvest and decreases with each subsequent passage. Corresponding with the increase in vimentin-positive cells over time the percent of epithelial/vimentin cells is highest at passage 1 but is absent in passage 2 . Values are mean percent \pm s.e.m.
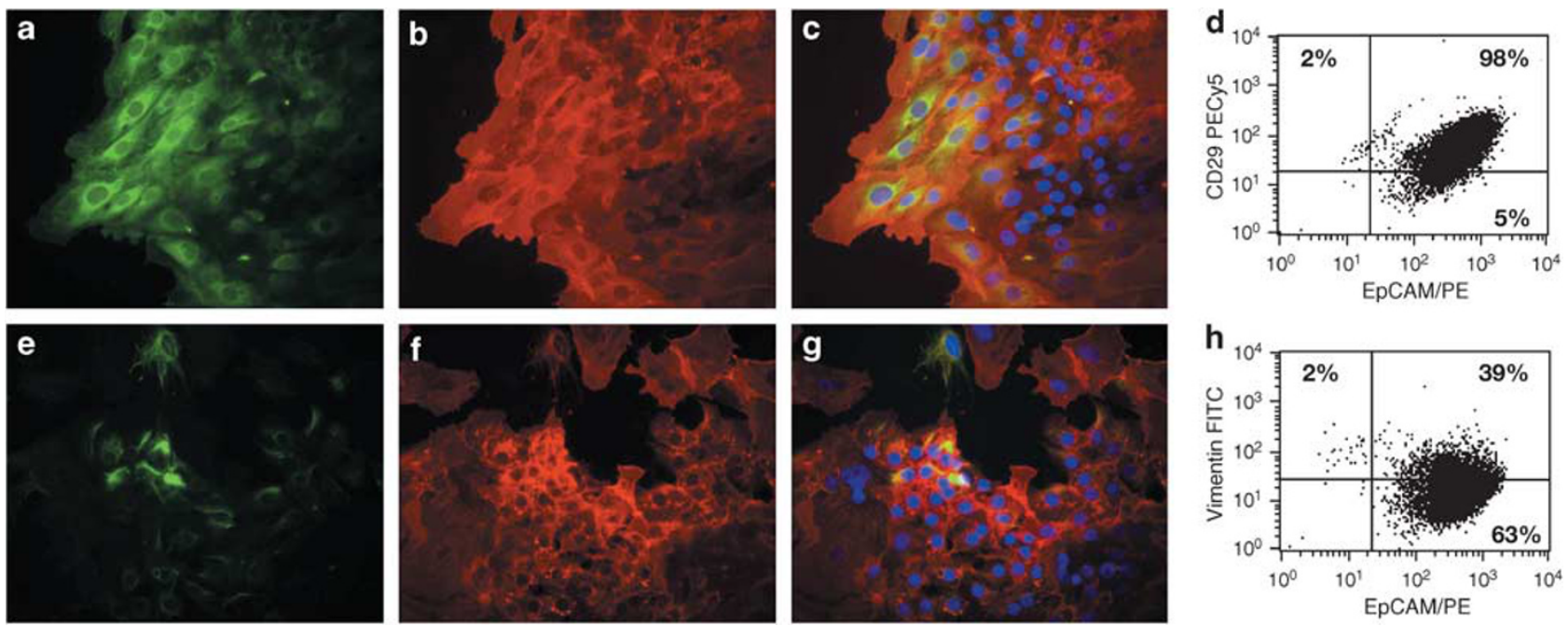

Figure 7 Nonendocrine pancreas expanded for $48 \mathrm{~h}$ on chamber slides contains epithelial cells that coexpress MSC antigens CD29 (a) and vimentin (e). Epithelial cells positive for EpCAM (red; $\mathbf{b}$ and $\mathbf{f}$ ) coexpress CD29 (c merged) and vimentin (g merged). Scatter plots (d and $\mathbf{h}$ ) demonstrate that the majority of EpCAM-positive cells are double-positive for CD29 (98\%) and a smaller percent of EpCAM-positive cells coexpress for vimentin (39\%) at this time point. Magnification is $400 \times$.

As in studies with bone marrow-derived MSCs, the isolation procedure itself may introduce genetic changes that affect cell phenotype and differentiation potential ${ }^{35,36}$ which may explain why epithelial cells coexpressed MSC antigens in the 2-day cultured pancreatic digest. Similarly, differences in donor characteristics, isolation or culture protocols and methods used to accurately define the cell composition of the mesenchymal progenitors ${ }^{20-23}$ and differentiated tissue could reflect the disparity seen between studies involving pancreatic mesenchymal progenitors. ${ }^{20-23}$

Following our differentiation procedure, we demonstrated that although we alter the MSC phenotype and our differentiated cells express mRNA transcripts for endocrine hormones and developmental factors ${ }^{20}$ we have no evidence that these are functional $\beta$-cells. We hypothesize that the low number of epithelial cells ( $<7 \%$; Figure 8$)$ we observed following differentiation may to contribute to our positive RT-PCR results (not shown). In addition, although CD44 is almost ablated (Figure 8); a large proportion of undifferentiated cells such as vimentin (91\%), CD90 (89\%) and CD29 (56\%) positive cells are present after differentiation, which indicates that differentiation is incomplete.

Unlike Davani et $a^{23}$ who suggest that they derive epithelial cell clusters (ECC) that are $\beta$-cell-like from mesenchymal precursors because these ECC have a small increase for transcripts of epithelial junctional proteins, 

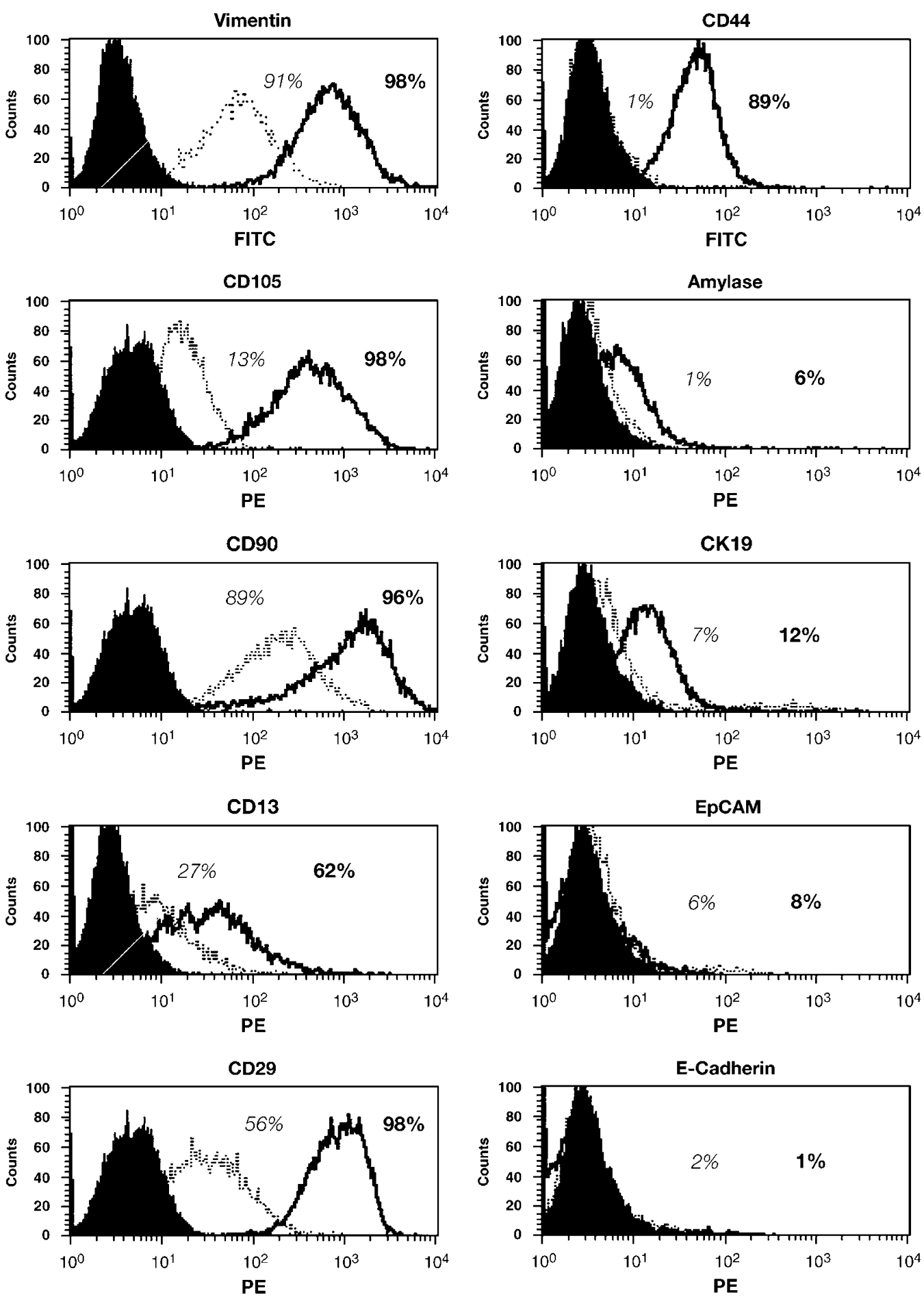

Figure 8 Representative MSC antigen profile of differentiated MSCs compared with undifferentiated MSCs. Following differentiation (solid gray line, italic script), CD44 expression is absent whereas the expression of other MSC antigens is decreased compared to undifferentiated MSCs (solid black line, bold script). CK19, EpCAM and E-Cadherin are equal to or less than $12 \%$. Solid histogram denotes isotype control.

most other studies have not truly assessed epithelial characteristics (ie, expression of EpCAM and E-Cadherin) of their differentiated cell populations. ${ }^{21,22}$ In our study, similar to mRNA transcripts for transcription factors and endocrine hormones we also detected transcripts for E-Cadherin (not shown) but detected minimal protein expression of this gene product $(<2 \%$; Figure 8$)$ which is a major deficiency in our differentiation protocol. The lack of epithelial cell antigens, especially E-Cadherin in our end product could explain why we do not detect insulin and may explain why in other 
studies cellular insulin levels are very low $(0.56 \%$ that of normal islets) and not secreted in response to glucose..$^{21,22}$

Several studies have recently demonstrated that cell adhesion via E-Cadherin regulates islet development, intercellular communication between $\beta$-cells and insulin secretion. ${ }^{37-39}$ In the developing mouse pancreas, Dahl et $a l^{37}$ demonstrated that disruption of E-Cadherin in $\beta$-cells inhibited the aggregation of endocrine cells into islet-like structures. In addition, when E-Cadherin was neutralized via antibody blockade, cell adhesion was lost and insulin secretion was reduced in MIN6 pseudoislets. ${ }^{38}$ Therefore, one must first determine whether MSCs can indeed differentiate into functional epithelium that express cell adhesion molecules like E-Cadherin that mediate intercellular communication and insulin secretion when assessing and interpreting insulin or C-peptide release from derived cells.

The origin and contribution pancreatic MSCs have in generating new islets for transplantation remains controversial. Similar to citations describing the difficulty differentiating bone marrow-derived $\mathrm{MSCs},{ }^{35}$ we cannot assume that pancreatic MSCs would be devoid of controversy. In a review by Phinney et al, ${ }^{35}$ the authors state 'revealing the full differentiation potential of MSCs will not likely occur until the biology of the bona fide MSC is fully described'. Whether MSCs are derived from bone marrow, adipose, muscle, peripheral blood or pancreas this is a heterogeneous cell population with varied plasticity and differentiation potential. ${ }^{35}$ The inconsistencies with respect to MSC differentiation into epithelial tissue may simply reflect the variation in isolation procedures or indicate that the plasticity assigned to this cell population in vitro may not reflect in vivo properties. ${ }^{35}$ Several recent in vivo studies have demonstrated that MSCs may not physically contribute to tissue regeneration themselves but instead secrete soluble factors that mediate endogenous tissue repair and regeneration. ${ }^{40-42}$ In particular in a recent study conducted by Lee et $a{ }^{41},{ }^{41}$ human MSCs when infused into diabetic NOD/SCID mice engraft to damaged tissue but appeared not to differentiate into new $\beta$-cells, but promoted the increase of endogenous islets and improved glomerular morphology. In a similar study, Hess et al ${ }^{40}$ reported that infused bone marrow cells engrafted to damaged pancreatic tissue resulting in endogenous pancreatic tissue regeneration and reduction of hyperglycemia but without expressing molecular factors associated with $\beta$-cell development. These and other studies suggest that MSCs may mediate tissue regeneration via endothelial interactions or by secretion of soluble factors in vivo. ${ }^{41,42}$ Therefore, the possibility exists that pancreatic MSCs that originate via EMT or preexist in islets/ pancreas may not have the potential to differentiate into a functional $\beta$-cell.

\section{ACKNOWLEDGEMENTS}

Funding for this project was provided by, grants from the Canadian Institutes of Health Research MOP 8030 and the Juvenile Diabetes Research
Foundation 5-2006-372. GSK is a senior scholar of the Alberta Heritage Foundation for Medical Research. We thank the staff of the Clinical Islet Laboratory (University of Alberta) as well as the HOPE program.

1. Ryan EA, Paty BW, Senior PA, et al. Five-year follow-up after clinical islet transplantation. Diabetes 2005;54:2060-2069.

2. Street CN, Lakey JR, Shapiro AM, et al. Islet graft assessment in the Edmonton Protocol: implications for predicting long-term clinical outcome. Diabetes 2004;53:3107-3114.

3. Korsgren $\mathrm{O}$, Lundgren $\mathrm{T}$, Felldin $\mathrm{M}$, et al. Optimising islet engraftment is critical for successful clinical islet transplantation. Diabetologia 2008;51:227-232.

4. Wang RN, Kloppel G, Bouwens L. Duct- to islet-cell differentiation and islet growth in the pancreas of duct-ligated adult rats. Diabetologia 1995;38:1405-1411.

5. Bonner-Weir S, Taneja M, Weir GC, et al. In vitro cultivation of human islets from expanded ductal tissue. Proc Natl Acad Sci USA 2000;97:7999-8004.

6. Heremans $Y$, Van De Casteele $M$, in't Veld $P$, et al. Recapitulation of embryonic neuroendocrine differentiation in adult human pancreatic duct cells expressing neurogenin 3. J Cell Biol 2002;159:303-312.

7. D'Alessandro JS, Lu K, Fung BP, et al. Rapid and efficient in vitro generation of pancreatic islet progenitor cells from nonendocrine epithelial cells in the adult human pancreas. Stem Cells Dev 2007:16:75-89.

8. $\mathrm{Xu} \mathrm{X}, \mathrm{D}^{\prime}$ Hoker J, Stange $\mathrm{G}$, et al. Beta cells can be generated from endogenous progenitors in injured adult mouse pancreas. Cell 2008;132:197-207.

9. Yatoh $S$, Dodge $R$, Akashi $T$, et al. Differentiation of affinity-purified human pancreatic duct cells to $\beta$-cells. Diabetes 2007;56:1802-1809.

10. Zhao M, Amiel SA, Christie MR, et al. Evidence for the presence of stem cell-like progenitor cells in human adult pancreas. J Endocrinol 2007;195:407-414.

11. Hao $E$, Tyrberg $B$, Itkin-Ansari $P$, et al. Beta-cell differentiation from nonendocrine epithelial cells of the adult human pancreas. Nat Med 2006;12:310-316.

12. Rooman I, Lardon J, Bouwens L. Gastrin stimulates beta-cell neogenesis and increases islet mass from transdifferentiated but not from normal exocrine pancreas tissue. Diabetes 2002;51:686-690.

13. Baeyens L, De Breuck S, Lardon J, et al. In vitro generation of insulinproducing beta cells from adult exocrine pancreatic cells. Diabetologia 2005:48:49-57.

14. Minami K, Okano H, Okumachi A, et al. Role of cadherin-mediated cellcell adhesion in pancreatic exocrine-to-endocrine transdifferentiation. J Biol Chem 2008;283:13753-13761.

15. Dor Y, Brown J, Martinez Ol, et al. Adult pancreatic beta-cells are formed by self-duplication rather than stem-cell differentiation. Nature 2004;429:41-46.

16. Teitelman G. Islet-derived multipotential cells/progenitor cells. Cell Biochem Biophys 2004;40:89-102.

17. Zulewski H, Abraham EJ, Gerlach MJ, et al. Multipotential nestinpositive stem cells isolated from adult pancreatic islets differentiate ex vivo into pancreatic endocrine, exocrine, and hepatic phenotypes. Diabetes 2001;50:521-533.

18. Gershengorn MC, Hardikar AA, Wei C, et al. Epithelial-to-mesenchymal transition generates proliferative human islet precursor cells. Science 2004:306:2261-2264.

19. Linning KD, Tai MH, Madhukar BV, et al. Redox-mediated enrichment of self-renewing adult human pancreatic cells that possess endocrine differentiation potential. Pancreas 2004;29:e64-e76.

20. Seeberger KL, Dufour JM, Shapiro AM, et al. Expansion of mesenchymal stem cells from human pancreatic ductal epithelium. Lab Invest 2006;86:141-153.

21. Eberhardt M, Salmon P, von Mach MA, et al. Multipotential nestin and Isl-1 positive mesenchymal stem cells isolated from human pancreatic islets. Biochem Biophys Res Commun 2006;345:1167-1176.

22. Gallo R, Gambelli F, Gava B, et al. Generation and expansion of multipotent mesenchymal progenitor cells from cultured human pancreatic islets. Cell Death Differ 2007;14:1860-1871.

23. Davani B, Ikonomour L, Raaka BM, et al. Human islet-derived precursor cells are mesenchymal stromal cells that differentiate 
and mature to hormone-expressing cells in vivo. Stem Cells 2007;25:3215-3222.

24. Lee KD, Kuo TK, Whang-Peng J, et al. In vitro hepatic differentiation of human mesenchymal stem cells. Hepatology 2004;40:1275-1284.

25. Seo MJ, Suh SY, Bae YC, et al. Differentiation of human adipose stromal cells into hepatic lineage in vitro and in vivo. Biochem Biophys Res Commun 2005;328:258-264.

26. Timper K, Seboek D, Eberhardt M, et al. Human adipose tissue-derived mesenchymal stem cells differentiate into insulin,somatostatin, and glucagon expressing cells. Biochem Biophys Res Commun 2006;341:1135-1140.

27. Battula VL, Bareiss PM, Treml S, et al. Human placenta and bone marrow derived MSC cultured in serum-free, b-FGF-containing medium express cell surface frizzled-9 and SSEA- 4 and give rise to multilineage differentiation. Differentiation 2007;75:279-291.

28. Tang $\mathrm{DQ}, \mathrm{Cao} \mathrm{LZ}$, Burkhardt $\mathrm{BR}$, et al. In vivo and in vitro characterization of insulin-producing cells obtained from murine bone marrow. Diabetes 2004;53:1721-1732.

29. Oh SH, Muzzonigro TM, Bae SH, et al. Adult bone marrow-derived cells trans-differentiating into insulin-producing cells for the treatment of type I diabetes. Lab Invest 2004;84:607-617.

30. Kayali AG, Flores LE, Lopez AD, et al. Limited capacity of human adult islets expanded in vitro to redifferentiate into insulin-producing betacells. Diabetes 2007;56:703-708.

31. Atouf F, Park $\mathrm{CH}$, Pechhold $\mathrm{K}$, et al. No evidence for mouse pancreatic beta-cell epithelial-mesenchymal transition in vitro. Diabetes 2007;56:699-702.

32. Chase LG, Ulloa-Montoya F, Kidder BL, et al. Islet-derived fibroblast-like cells are not derived via epithelial-mesenchymal transition from $\mathrm{Pdx}-1$ or insulin-positive cells. Diabetes 2007;56:3-7.
33. Morton RA, Geras-Raaka E, Wilson LM, et al. Endocrine precursor cells from mouse islets are not generated by epithelial-to-mesenchymal transition of mature beta cells. Mol Cell Endocrinol 2007;270:87-93.

34. Russ HA, Bar Y, Ravassard $P$, et al. In vitro proliferation of cells derived from adult human $\beta$-cells revealed by cell-lineage tracing. Diabetes 2008;57:1575-1583.

35. Phinney DG, Prockop DJ. Concise review: mesenchymal stem/ multipotent stromal cells: the state of transdifferentiation and modes of tissue repair-current views. Stem Cells 2007;25:2896-2902.

36. Marselli L, Thorne J, Ahn Y, et al. Gene expression of purified b-cell tissue obtained from human pancreas with laser capture microdissection. J Clin Endocrinol Metab 2008;93:1046-1053.

37. Dahl U, Sjödin A, Semb H. Cadherins regulate aggregation of pancreatic $\beta$-cells in vivo. Development 1996;122:2895-2902.

38. Rogers GJ, Hodgkin MN, Squires PE. E-Cadherin and cell adhesion; a role in architecture and function in the pancreatic islet. Cell Physiol Biochem 2007;19:987-994.

39. Bosco D, Rouiller DG, Halban PA. Differential expression of E-cadherin at the surface of rat $\beta$-cells as a marker of functional heterogeneity. J Endocrinol 2007;194:21-29.

40. Hess D, Li L, Martin M, et al. Bone marrow-derived stem cells initiate pancreatic regeneration. Nat Biotechnol 2003;21:763-770.

41. Lee RH, Seo MJ, Reger RL, et al. Multipotent stromal cells from human marrow home to and promote repair of pancreatic islets and renal glomeruli in diabetic NOD/scid mice. Proc Natl Acad Sci USA 2006;103:17438-17443.

42. Iso $\mathrm{Y}$, Spees $\mathrm{JL}$, Serrano $\mathrm{C}$, et al. Multipotent human stromal cells improve cardiac function after myocardial infarction in mice without long-term engraftment. Biochem Biophys Res Commun 2007;354:700-706 